\title{
Novel Development of Corrosion Resistant Paint Using Printed Circuit Board from Modern Electronic Wastes
}

\author{
Mohana Rajendran* and Marto Giftlin \\ Department of Civil Engineering, Mepco Schlenk Engineering College, Sivakasi, India
}

('Corresponding author's e-mail: rmohana@mepcoeng.ac.in)

Received: 13 October 2020, Revised: 2 August 2021, Accepted: 13 August 2021

\begin{abstract}
The growth in the industrial technology and communication sectors has enhanced the usage of electronic gadgets exponentially. Thus, it becomes highly essential to find new solution to utilize or dispose these e-wastes, in order to reduce the e-waste generation. Various reports mentioned that the major component of these e-wastes is their Printed Circuit Boards. Therefore, a new ideology is proposed to use these boards to produce a new coating with good corrosion resistance. At first the boards were separated from the discarded electronic appliances and various electrical elements were separated from these boards. Then the plain circuit boards are pulverized into fine particles and mixed with paint in 3 different proportions ranging from 1:4 to 1:2. The resulting mix was applied over the steel specimen and tested for corrosion resistance. The assessment was done in the aspects of contact angle, weight loss percentage, potentio dynamic polarization technique and electro chemical impedance spectroscopy. From the results it was clear that increase in the addition of printed circuit powder content in the corrosion proof paint coating enhanced the efficiency of the corrosion resistant behaviour 3.5 times than the specimen with uncoated steel specimens. This development of corrosion resistant behaviour of printed circuit powder was attributed from the inertness offered by the presence of silicon dioxides towards the corrosive fluids. Although, the coating of 1:2 proved to be optimum to protect the steel elements from the aggressive corrosion environment, by considering the workability aspects the 1:3 combination was suggested for the innovative development of anti-corrosion paint coating from the e-waste resources.
\end{abstract}

Keywords: E-waste generation, Corrosion resistance, Potentiodynamic polarization, Printed circuit board, Discarded electronic appliances, Plain circuit board

\section{Introduction}

Along the Indian timeline waste generation has created various socio-economic hazards. India ranks fifth among the world's electronic gadget producers. On an average India produces nearly 2 million tons of e-waste annually. In addition to this India has been leading the line in terms of import of electronic goods. Annually, computer devices such as television sets and computers contribute about $70 \%$ of ewaste, telecom sector has been an influential contributor with a contribution of $20 \%$ and both medical and electrical industries contributing 8 and $6 \%$ respectively. The government and the separate business sectors including public and private had been brimming with their electronic waste production at $75 \%$ with the major contribution coming from individual household claimed at $12 \%$. Most of these products can be processed under the 3R's namely Reuse, Reduce and Recycle. Regular evolution of electronic gadgets has produced new items like smartphone, tablets, laptops, video game consoles, cameras and many more. At the end of 2018, India had an astounding 1.018 billion mobile service connection which may lead to the increase of e-waste into the solid stream. These numbers do not seem to diminish but on the other hand, it goes on increasing exponentially [1]. ASSOCHAM, is an industrial body in India which makes surveys on the generation of e-wastes revealed that, Compound Annual Growth Rate (CAGR) of electronic waste is $30 \%$. With people showing bad attitude towards waste management and worrying consumer behavior ASSOCHAM predicts that India will generate about 5.2 tons of electronic waste. It has been found that the major component in the electronic gadget is their circuit boards.

To establish an effective recycling process for waste appliances, the process of recovering printed circuit boards (PCBs) containing valuable elements in comminution was investigated and evaluated. The present study performed comminution tests using 3 different types of waste appliances: Smartphones, microwave ovens and electrical rice cookers. Comminution tests showed that a drum-type agitation mill 
operated at a mid-range rotation speed could achieve a relatively high recovery ratio of PCBs and inhibit excessive breakage of PCBs. Following these experiments, simulations using the discrete element method with a particle-based rigid-body model were conducted to evaluate the comminution performance of the drum-type agitation mill. Experimental and simulation results confirm that the processes of detachment of PCBs from waste appliances and subsequent breakage can be expressed by kinetic equations related to collision energy. It is concluded from these results that the kinetic equations obtained in experiments and simulations can be used to evaluate the recovery process of PCBs from waste appliances[2].Printed circuit boards (PCBs) from discarded personal computer (PC) and hard disk drive were crushed by explosion in water or mechanical comminution in order to disintegrate the attached parts. More parts were stripped from PCB of PC, composed of epoxy resin; than from PCB of household appliance, composed of phenol resin [3].

PCB generally consists of 4 layers, which are heat laminated together into 1 layer. The fabric utilized in PCB from top to bottom includes Silkscreen, Soldermask, Copper and Substrate. The last of these layers, substrate, is formed of fiberglass and is additionally cited as FR4, with the FR letters standing for "fire retardant". This substrate layer provides a solid foundation for PCBs, though the thickness can vary in line with the uses of a given board. A cheaper range of boards also exist on the market that do not utilize the identical aforementioned PCB materials, but instead contains phenolics or epoxies. Because of the thermal sensitivity of those boards, they have an inclination to lose their lamination easily. These cheaper boards are often easy to spot by the smell they furnish off when being soldered. PCB second layer is copper, which is laminated onto the substrate with a combination of warmth and adhesive. The copper layer is thin, and on some boards, there are 2 such layers - 1 above and 1 below the substrate. PCBs with only 1 layer of copper tend to be used for cheaper electronics devices. The massively used copper clad laminate (CCL) are often classified into different categories in line with different classification standards including reinforcing material, used resin adhesive, flammability, CCL performance [4]. Table 1 shows the composition of PCB.

Table 1 Composition of printed circuit boards [18].

\begin{tabular}{ccc}
\hline Metal & OREs (\%) & PCBs (\%) \\
\hline Copper & $0.5-3.0$ & $12.0-29.0$ \\
Zinc & $1.7-6.4$ & $0.1-2.7$ \\
Tin & $0.2-0.85$ & $1.1-4.8$ \\
Lead & $0.3-7.5$ & $1.3-3.9$ \\
Iron & $30-60$ & $0.1-11.4$ \\
Nickel & $0.7-2.0$ & $0.3-1.6$ \\
Gold & 0.0005 & $0.0029-0.112$ \\
Silver & 0.0005 & $0.01-0.52$ \\
\hline
\end{tabular}

Millions of tons of electronic wastes were dumped without knowing the ways to dispose which affects the environment and human society. The rate of recycling and reusing the printed circuit boards are very less when compared to the disposal rate. On this note, to enhance the e-waste management and also used the derived and recycled products to use, a new ideology of using Printed Circuit Boards as a source for producing a corrosion resistant coating is proposed in this paper. Creative utilization of waste printed circuit board powers for the development of anti-corrosion paint for the modern steel structures and elements were investigated first time in this paper. The main aim of this research work is to prove the applicability of electronic wastes for the newly developed corrosion proof paint for the protection steel members against corrosion effects. Hence a novel theme of research was invented to use the abundant printed circuit board powders for the corrosion protection paint development in order to achieve environmental sustainability against electronic waste generation of modern society and cost-effective durable protection against the harmful corrosive effects simultaneously. The assessment of corrosion resistance was done in the aspects of contact angle, percentage weight loss, polarization effect and the electro chemical spectroscopic analysis for the various mix proportion of printed circuit board powder from 1:4 to 1:3. The optimum mix composition was finalized by considering the aforementioned factors and workability from the experimental results as discussed in this paper. 


\section{Materials and methods}

\section{Coating preparation}

Removal of preliminary elements from PCB Boards

The PCB boards contains both target and non-target materials. Target materials includes copper, zinc, tin, etc. Non-target materials include transistors, resistors, batteries, capacitors and LEDs. Our main aim is to remove non-target materials. Initially, we removed the non-target materials manually with the help of cutting pliers. The remaining scrap materials were removed by de-soldering. For the removal of a green colored silicon layer in those boards, mask tapes were used. Mask tapes were made to stick on these boards and after removal it erodes the silicon layer on these boards [5].

\section{Desoldering}

Desoldering requires application of heat to the solder joint and removing the molten solder that the joint could even be separated. Desoldering could even be required to interchange a defective component, to vary an existing circuit, or to salvage components for re-use. Use of too high a temperature or heating for too long may damage components or destroy the bond between an electric circuit trace and also the board substrate. The non-target materials were soldered to the circuit boards. On manual removal of these non-target materials the scrap material will hold on to the boards. Thus, desoldering is done i.e.) the scrap material is heated to remove the bond and a desolder pump is used to pump out the scrap material [6]. The Figure 1 shows the board after desoldering with non-target components.

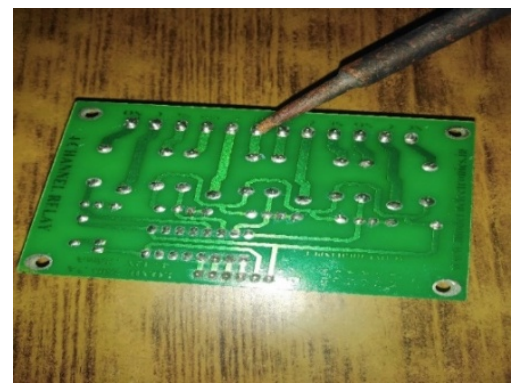

Figure1 Board with non-target components

\section{Immersion in $\mathrm{HCL}$}

Once the scrap materials were removed from the board using de-soldering, the board was then immersed in a plastic container having dilute HCL for about $8 \mathrm{~h}$ to remove target materials which were toxic as shown in the Figure 2. The HCl-mediated leaching of waste PCBs was investigated over a range of conditions. Increasing the acid concentration decreased the time required for complete metal recovery. By this process, the presence of lead, aluminum and nickel if any will be precipitated at the bottom of the container as black precipitate. The precipitate will be collected, and these fresh boards will be subjected to pulverization[7].

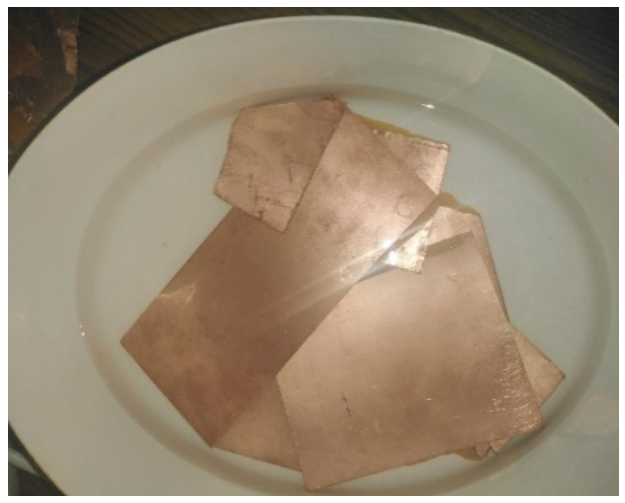

Figure 2 Boards immerse in HCL 


\section{Pulverization}

At first, the plain circuit boards were broken into pieces by the help of consolidation equipment. Once these boards were broken into fine fragments, these boards were sent to ball mills. In the ball mill, these fragments were operated on for 6 days at the rate of 4 ha day. At the end of the pulverization process $200 \mathrm{~g}$ of yellowish white powder of irregular shape was obtained as shown in theFigure 3. The powder emitted an epoxy smell confirming the presence of epoxy in the powder [8].

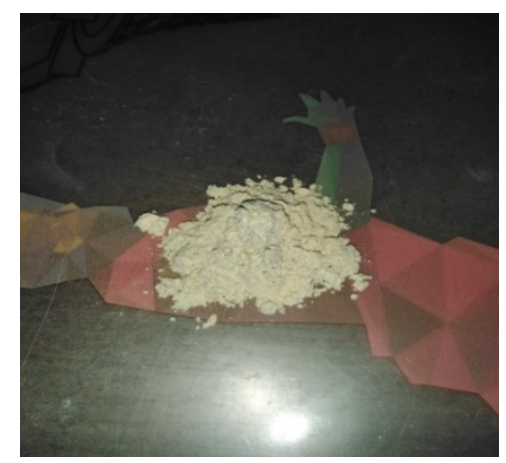

Figure 3PCB powder

\section{Coating process}

Threedifferent types of coating were prepared to be coated on the steel specimen for corrosion studies. There are no standard mix proportions for these coatings, so trial and error method were followed regarding the preparation of coatings [9]. The coatings are as follows:

1. Powder solution + Paint coating (1:2 mix proportion)

2. Powder solution + Paint coating ( 1:3 mix proportion)

3. Powder solution + Paint coating (1:4 mix proportion)

\section{Methods}

\section{Contact angle measurement}

Contact angle test was done to find the ability of inter molecular interactions present on the metal surfaces to resist the water adherence. The test was made by a distilled water drop on metal surface at 20 ${ }^{\circ} \mathrm{C}$. Based on the angle made by the water droplet on the specimen surface the hydrophobic film layer of the coated layer can be determined as shown in Figure 4. For a good hydrophobic film layer, the value should be between $80-120^{\circ}$. The contact angle of a specimen can be given by the YOUNG-DUPRE as given in Eq. (1).

$\gamma \mathrm{SG}-\gamma \mathrm{SL}-\gamma \mathrm{LG} \cos \theta \mathrm{c}=0$

where:

$\gamma \mathrm{SG}$ : Denote solid-vapor interfacial energy

$\gamma \mathrm{SL}$ : Denote solid-liquid interfacial energy

$\gamma$ LG: Denote liquid-vapor interfacial energy

$\theta c$ : Angle made by the water droplet

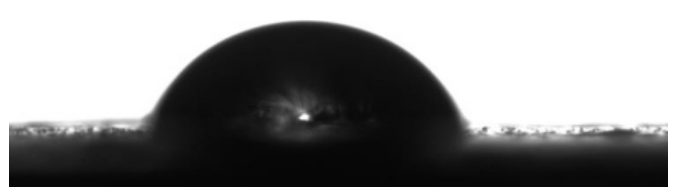

Figure 4 Contact angle measurement 
Optical tensiometer is an instrument used to find the contact angle, wettability and adsorption property of the material [10] whose technical specifications are shown in Table 2.

Table 2 Technical specification of optical tensiometer.

\begin{tabular}{cc}
\hline Measuring range & $0-180^{\circ}, 0.01-2000 \mathrm{mN} / \mathrm{m}$ \\
Accuracy & $\pm 0.1,0.01^{\circ} \mathrm{mN} / \mathrm{m}$ \\
Maximum sample size & $\mathrm{L} 200 \times \mathrm{H} 45 \mathrm{~mm}^{2}$ \\
Frame interval & $0.48 \mathrm{~ms}-1000 \mathrm{~s}$ \\
Resolution & $1280 \times 1024$ pixel \\
Max. measuring speed & 2068 frames \\
Camera & USB 3.0 digital camera \\
Light Source & LED based background light \\
Dimension & H $310 \mathrm{~mm}, \mathrm{~W} 130 \mathrm{~mm}, \mathrm{~L} 495 \mathrm{~mm}$ \\
Weight & $5 \mathrm{~kg}$ \\
Power supply & $100-240 \mathrm{VAC}$ \\
Frequency & $50-60 \mathrm{~Hz}$ \\
\hline
\end{tabular}

\section{Weight loss method}

This study was conducted to examine the corrosion behavior of steel specimen in acid solutions using weight loss technique. The magnitude of corrosion of these metals in $0.5-3.0 \mathrm{M}$ of solution of sulphuric acid was studied for an exposure period of $180 \mathrm{~min}$ (at half-hour interval) as shown in Figure 5.

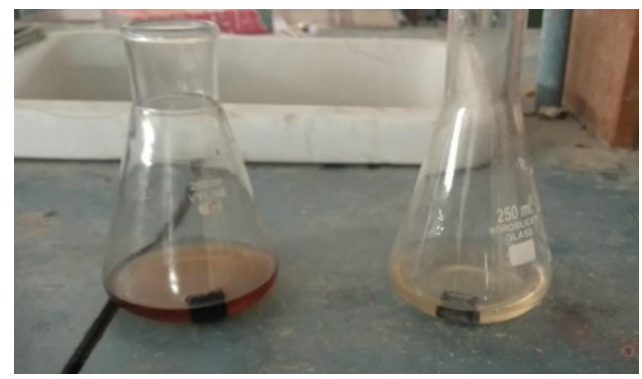

Figure 5 Flask containing specimen and reagents

\section{Rate of corrosion in metals followed this order:}

Zinc $>$ Galvanized iron $>$ Copper.

\section{Rate of corrosion in aqueous media followed this order:}

$\mathrm{HNO} 3>\mathrm{H} 2 \mathrm{SO} 4>\mathrm{HCl}$.

It has been obtained that increase in the concentration leads to increase in rate of corrosion [11]and the properties of suphuric acid are given inFigure 6. It is attributed due to the shedding of nitrite, sulphate and chloride ions on the surface of the metals resulted in the detrimental formation of magnetite compounds and hematite compound. The oxygen atoms absorb the main rust products and leads to the loss of adherence to the metal surface and protectiveness against corrosion [19].

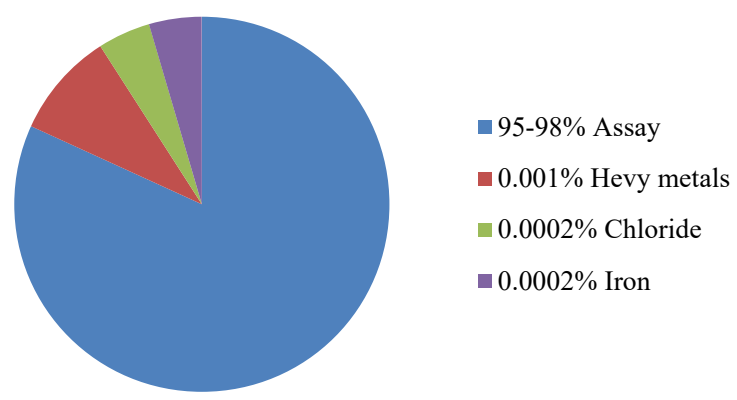

Figure 6 Composition of sulphuric acid used in the test 


\section{Electrochemical Impedance Spectroscopy (EIS)}

A vital gain of EIS over 1-of-a-kind laboratory strategies is the opportunity of the usage of very small amplitude signals without drastically traumatic the houses being measured. To make an EIS dimension, a small amplitude sign, commonly a voltage among 5 to $50 \mathrm{mV}$, is applied to a specimen over a number of frequencies of 0.001 to $100,000 \mathrm{~Hz}$.
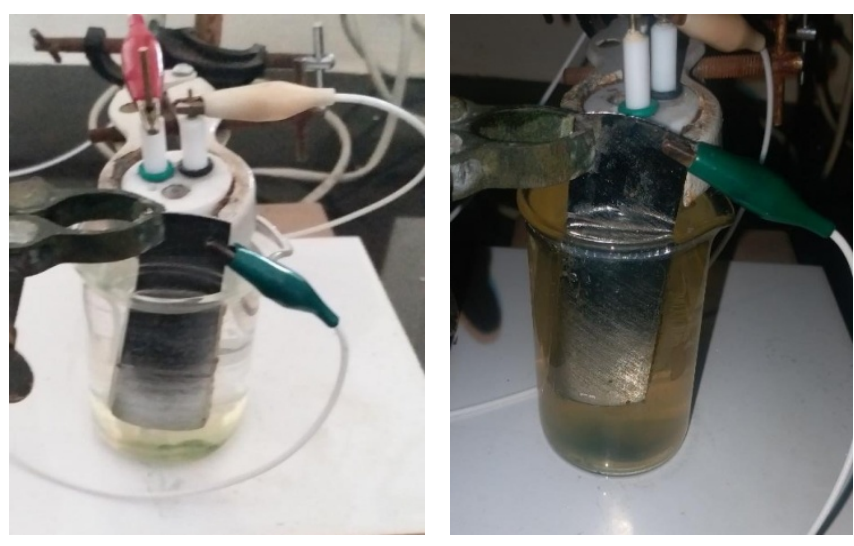

Figure 7Electrochemical test apparatus

$3.5 \mathrm{gm}$ of Sodium Chloride powder was taken in a spatula and mixed with $100 \mathrm{~mL}$ of dilution water. The specimen was held vertically and immersed in the electrolyte solution as shown in Figure7. After the preliminary setup of the apparatus is done, current supply is given and emf is induced. Based on the emf induced, the coated plates are also subjected to corrosive environment and observations are made which is plotted in TAFEL graph.

\section{Results and discussion}

The recovered printed circuit board powder was blended with an adhesive agent i.e.,paint, in 3 specific proportions based on the trial-and-error method. The efficiency of the corrosion resistant coating was observed from the results of 4 renowned electrochemical tests and the results were plotted in a graph.

\section{Contact angle measurement}

From theTable 3, it is evident that, as the amount of printed circuit board powder in the mix increases the contact angle also increases thus reducing its affinity towards water and other corrosive fluids, which in turn increases the corrosion resistive efficiency of the surface. If the concept is explained more precisely, the contact angle greater than 90 degree are less prone to hydrophobic effects thus making it less prone to corrosion. The development of super hydro phobicity is mainly attributed from the cumulative interactions of the highly cross-linked covalent bonding of printed circuit board powders on the metal surface and the water molecules [13].In this case 2 proportions produced results with contact angle greater than 90 degree. The most efficient coating observed is 1:2 mix, but for better workability and fluidity 1:3 mix is suggested because the difference in results of both the mixes is meager.

Table 3Results of contact angle measurements

\begin{tabular}{cc}
\hline Specimen & Contact angle observation \\
\hline Bare specimen & $74^{\circ}$ \\
Specimen with 1:4 & $89^{\circ}$ \\
Specimen with 1:3 & $92^{\circ}$ \\
Specimen with 1:2 & $97^{\circ}$ \\
\hline
\end{tabular}




\section{Weight loss method}

From the Table4, it is evident that, as the amount of printed circuit board powder in the mix increases the weight loss in the specimen reduces thus reducing its affinity towards water and other corrosive fluids, which in turn increases the corrosion resistive efficiency of the surface. If the concept is explained more precisely, the printed circuit board which comprises of silicon dioxide particles pragmatically becomes inert to corrosive substances. Thus, more the PCB powder more the corrosion resistance but again accounting for fluidity and workability regarding the mix thus 1:3 mix is preferred over 1:2 mix although their results are in the same range.

Table 4 Results of weight loss technique

\begin{tabular}{ccccc}
\hline Sample & Bare (g) & $\begin{array}{c}\mathbf{1 : 2} \text { Mix proportion } \\
(\mathbf{g})\end{array}$ & $\begin{array}{c}\mathbf{1 : 3} \text { Mix proportion } \\
(\mathbf{g})\end{array}$ & $\begin{array}{c}\mathbf{1 : 4} \text { Mix proportion } \\
\text { (g) }\end{array}$ \\
\hline $\mathrm{W}_{\mathrm{o}}$ (Initial) & 9.397 & 9.861 & 9.906 & 9.915 \\
$\mathrm{~W}_{\mathrm{f}}($ Final) & 9.311 & 9.851 & 9.901 & 9.912 \\
$\mathrm{CR} \mathrm{Avg}\left(10^{-4} \mathrm{~mm} / \mathrm{h}\right)$ & 5.89 & 3.31 & 3.28 & 3.07 \\
\hline
\end{tabular}

$\mathrm{W}=9.756-9.755=0.001 \mathrm{~g}, \mathrm{~K}=534, \mathrm{D}=7850 \mathrm{~g} / \mathrm{cm}^{3}, \mathrm{~A}=50.24 \mathrm{~mm}^{2}, \mathrm{t}=0.5 \mathrm{~h}, \mathrm{CR}=5.88 \times 10^{-4}(\mathrm{~mm} / \mathrm{h})$

\section{Potentio-dynamic polarization}

From the Table 5, we can interpret the values of 2 terms namely, Corr I (corrosion coefficient) and rate of corrosion. If the value of corrosion coefficient is high, it infers that the surface is highly prone to corrosion and if the rate of corrosion is high, it also reveals that the surface is highly prone to deterioration by corrosive fluids. If the corrosion prone fluid medium was get contact with the steel specimen the immediate action is the conversion of ferrous compound into ferric oxide compounds which is the initiative of rust formation [14]. This rate of formation of ferric oxide compounds purely depends on the preexisting holes available for the electrolyte percolation. If these corrosive pore domains are coated with the heterogeneous rough atoms like nitrogen or silica, the rate of ferrous oxide production will be highly reduced [15]. In accordance with the increase of printed circuit board powder in the mixture, the corrosion resistance efficiency of the coating increases. Again, the best results were obtained for the 1:2 mix proportion but for fluidity concerns 1:3 mix is suggested, as the results of both mixes are in the near range. Further this coating is 3 times more effective than a surface without a corrosion protection and a renowned corrosion inhibitor in said to be 3.5 times more effective than a surface without protection, thus satisfying our requirement to be used as a corrosion resistant coating.

Table 5 Observed readings of PDP test.

\begin{tabular}{ccccc}
\hline Sample & $\begin{array}{c}\text { Bare } \\
\text { (without coating) }\end{array}$ & $1: 4$ Proportion & $1: 3$ Proportion & $1: 2$ Proportion \\
\hline CAT slope(I/V) & 3.600 & 6.172 & 5.676 & 5.927 \\
ANO slope(I/V) & 9.315 & 4.290 & 4.656 & 4.565 \\
CAT int(log i) & -3.325 & -3.701 & -3.671 & -3.693 \\
ANO int(log i) & -2.870 & -3.698 & -3.545 & -3.613 \\
Lin Pol R(ohm) & 37 & 148 & 159 & 153 \\
CORR I (A) & $9.136 \mathrm{e}-0.004$ & $2.816 \mathrm{e}-0.002$ & $2.398 \mathrm{e}-0.002$ & $2.586 \mathrm{e}-0.002$ \\
Rate $(\mathrm{mm} / \mathrm{year})$ & $4.169 \mathrm{e}+0.002$ & $1.286 \mathrm{e}+0.002$ & $1.342 \mathrm{e}+0.002$ & $1.319 \mathrm{e}+0.002$ \\
Rate $($ Angs $/ \mathrm{min})$ & $2.014 \mathrm{e}+0.002$ & $5.214 \mathrm{e}+0.01$ & $5.780 \mathrm{e}+0.01$ & $5.931 \mathrm{e}+0.01$ \\
Rate $(\mathrm{gram} / \mathrm{h})$ & $9.518 \mathrm{e}-0.004$ & $2.934 \mathrm{e}-0.002$ & $3.012 \mathrm{e}-0.002$ & $2.96 \mathrm{e}-0.002$ \\
\hline
\end{tabular}


Electrochemical Impedance Spectroscopy (EIS)

The process of corrosion forced the output current to be out of phase of the input voltage. This ratio between the input voltage and the output current is known as impedance. This impedance spectroscopy technique is the direct assessment of corrosion rate on any metal surface based on the phase angle graphplotted between the input voltage to the output current known as TAFEL plot [16]. Figure 8 indicates the TAFEL plot or the dip in the emf induced with directly indicates the corrosion resistance of the specimen. Samples with an early tafel or an early dip in the emf plot indicates that the specimen will corrode early when compared to other specimens. The delay in the dip or tafel can be attributed to the voltage applied. It takes a high voltage for the electrochemical reaction to take place for the completion of corrosion process. The reason behind this is the incorporation of printed circuit powders absorbs the hydrogen and chlorine atoms and already absorbed water molecules by the metal surfaces [17]. This rate of absorption of chemical products by the printed circuit powder coating delayed the metal surface to react with the external agents and thus the shift of curve takes place from -0.65 to $-0.55 \mathrm{~V}$. It that case, the 1:2 mix showed greater delay in tafel, thus making it highly resistant. But for fluidity and smoothness of the surface the 1:3 mix is suggested as it also has a good delayed response for the corrosion reaction.

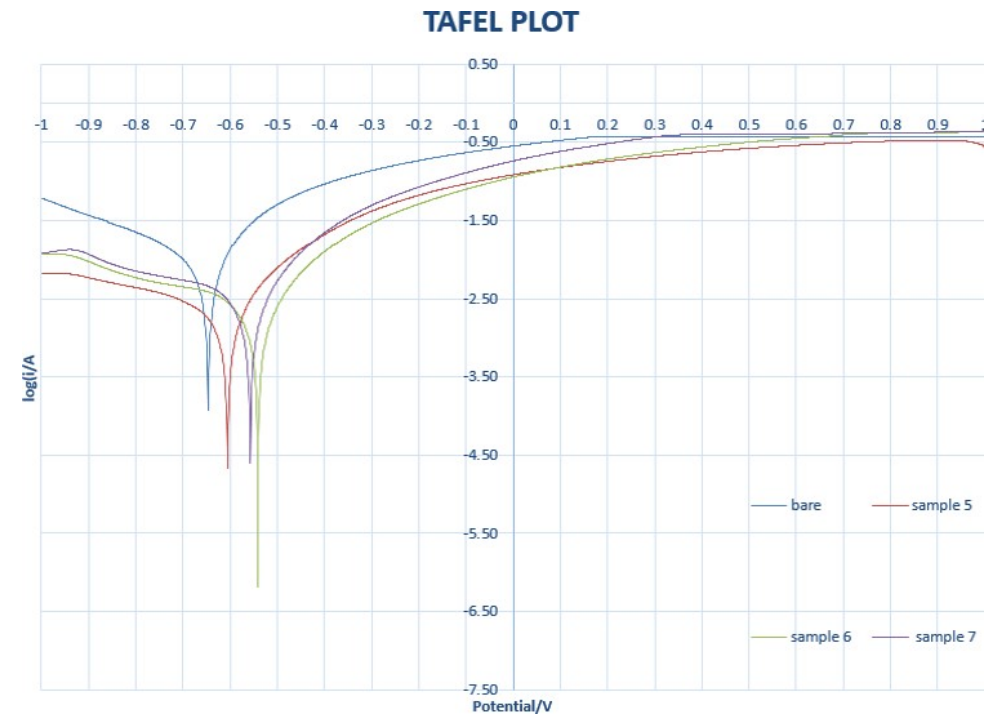

Figure 8 Tafel plot graph (sample 5 -proportion 1:3, sample 6 - proportion 1:4 and sample 7 - proportion $1: 2)$

\section{Conclusions}

The paper had come with a new E-waste management technique that had led to the discovery of a new corrosion resistant coating. It is concluded that the corrosion resistant efficiency is high when the printed circuit board powder is mixed at a proportion of 1:3. The coating using printed circuit board powder has been proved to be resistant against sulphate attack, chloride attack as these coatings were tested against these electrolytes. In addition to corrosion resistance these coatings also add an aesthetic value producing a shiny appearance. The coated steel bars can be used for reinforcing steel in reinforced concrete structures. Further, these coatings can be given to steel structures which is prone to corrosion.

\section{References}

[1] I Cherukuri, N Sultana and SP Podila. Status of e-waste in India: A review. J. Environ. Sci. Toxicol. Food Tech. 2018; 12, 8-16.

[2] Y Tsunazawa, S Hisatomi, S Murakami and CTokoro. Investigation and evaluation of the detachment of printed circuit boards from waste appliances for effective recycling. Waste Manag. 2018; 78, 474-82.

[3] T Fujita, H Ono, G Dodbiba and K Yamaguchi. Evaluation of a recycling process for printed circuit board by physical separation and heat treatment. Waste Manag. 2014; 34, 1264-73. 
[4] A Hubau, A Chagnes, M Minier, S Touzé, S Chapron and AG Guezennec. Recycling-oriented methodology to sample and characterize the metal composition of waste printed circuit boards. Waste Manag. 2019; 91, 62-71.

[5] L Pietrelli, S Ferrobn and M Voccianted. Eco-friendly and cost-effective strategies for metals recovery from printed circuit boards. Renew. Sustain. Energ. Rev. 2019; 112, 317-23.

[6] M Kaya. Waste printed circuit board (WPCB) recovery technology: Disassembly and desoldering approach. Encyclopedia Renew. Sustain. Mater. 2020; 4, 658-76.

[7] U Jadhav and H Hocheng. Hydrometallurgical recovery of metals from largeprinted circuit board pieces. Sci. Rep. 2015; 5, 14574.

[8] $\mathrm{H} \mathrm{Lu}, \mathrm{J} \mathrm{Li}, \mathrm{J}$ Guo and ZM Xu. Pulverization characteristics and pulverizing of waste printed circuit boards (printed wiring boards) based on resource utilization. J. Shanghai Jiaotong Univ. 2007; 41, 551-6.

[9] A Anthofer, P Kögler, C Friedrich, W Lippmann and A Hurtado. Laser decontamination and decomposition of PCB-containing paint. Optic Laser Tech. 2017; 87, 31-42.

[10] YM Liu, ZQ Wu and DC Yin. Measurement of contact angle under different gravity generated by a longarm centrifuge. Colloid Surface Physicochem. Eng. Aspect 2020; 588, 124381.

[11] AD Usman, AF Victoria, LN Okoro and N Linus. Weight loss corrosion study of some metals in acid medium. J. Adv. Chem. 2015; 11, 3435-40.

[12] RI Holland. Corrosion testing by potentiodynamic polarization in various electrolytes. Dent. Mater. 1992; 8, 241-5.

[13] Y Bao, Y Yan, J Zhang, J Ma, W Zhang and C Liu. Effect of the feeding mode of cross-linker and microcapsule on the corrosion resistance and hydrophobicity of composite coatings. Arabian J. Chem. 2020; 13, 9068-80.

[14] W Tian, F Meng, L Liu, Y Li and F Wang. The failure behaviour of a commercial highly pigmented epoxy coating under marine alternating hydrostatic pressure. Progr. Org. Coating 2015; 82, 101-12.

[15] E Javadia, M Ghaffari, $G$ Bahlakeh and $P$ Taheri. Photocatalytic, corrosion protection and adhesionproperties of acrylic nanocomposite coating containing silane treated nano zinc oxide: A combined experimental and simulation study. Progr. Org. Coating 2019; 135, 496-509.

[16] ZP Mathew, KRC Augustinea, B Joseph and S Johna. Corrosion inhibition of mild steel using poly (2-ethyl-2-oxazoline) in $0.1 \mathrm{M} \mathrm{HCl}$ solution author links open overlay panel. Heliyon 2020; 6, 05560 .

[17] D Borisova, D Akçakayiran, M Schenderlein, H Möhwald and DG Shchukin. Nanocontainer-based anticorrosive coatings: Effect of the container size on the self-healing performance. Adv. Funct. Mater. 2013; 23, 3799-812.

[18] WA Bizzo, RA Figueiredo and VF de Andrade. Characterization of printed circuit boards for metal and energy recovery after milling and mechanical separation. Materials 2014; 7, 4555-66.

[19] G Priyotomo. A field study of atmospheric corrosion of carbon steel after short exposure in Pelabuhan Ratu, West Java Province, Indonesia. Walailak J. Sci. Tech. 2021, 18, 9667. 